\title{
Ultra-high-energy cosmic rays by Cygnus A or the bulk of non-local radio galaxies?
}

\section{Björn Eichmann*}

Ruhr Astroparticle and Plasma Physics Center (RAPP Center), Ruhr-Universität Bochum, Institut für Theoretische Physik IV, 44780 Bochum, Germany

E-mail: eiche@tp4.rub.de

The great potential that radio galaxies are the birthplace of ultra-high-energy cosmic rays (UHECRs) has been shown by numerous works so far. In addition to the close-by Fanaroff and Riley (FR) radio galaxy Centaurus A that most likely provides the dominant UHECR contribution at the highest energies, there is an other contributor between $5 \mathrm{EeV}$ and $30 \mathrm{EeV}$ needed. The exceptionally bright radio source Cygnus $\mathrm{A}$ is a prominent source candidate, but it is shown in this work, that the impact by the extragalactic magnetic field on the CR propagation causes some serious issues: Either the arrival directions of the CRs provide a high degree of anisotropy or the delay exceeds the source age. Alternatively, the low-energetic UHECRs can originate in the bulk of FRI or FR-II sources. For such a scenario, the necessary jet dynamics of FR sources are discussed, showing that FR-I RGs can in principle provide the observed amount of UHECR energy as well as a proper spectral behavior. In contrast, the bulk of FR-II RGs most likely contribute less than $25 \%$.

36th International Cosmic Ray Conference -ICRC2019-

July 24th - August 1st, 2019

Madison, WI, U.S.A.

${ }^{*}$ Speaker. 


\section{Introduction}

The chance to unveil the mystery of the ultra-high-energy cosmic rays (UHECRs) never seemed as feasible as it does today. On the observational side, there are high precision measurements from different observatories of their main properties, like the energy spectrum and the chemical composition up to about $100 \mathrm{EeV}$. In addition, the recent observations of a dipole anisotropy of the arrival directions with an amplitude of $\simeq 6.5 \%$ above $8 \mathrm{EeV}$ indicate the first imprints of an outstanding source direction at the highest energies. But since CRs are fully ionized nuclei, that show an increasing mass/ charge number above about $1 \mathrm{EeV}$, the impact of the Galactic and extragalactic magnetic fields prevents an identification of the sources.

Moreover, the extragalactic magnetic fields are poorly known, especially in the large-scale structures of the Universe. However, the computational evolution in recent years allowed for sophisticated descriptions of the extragalactic magnetic fields (EGMF) up to distances of more than $100 \mathrm{Mpc}$, e.g. [1,2]. There are two basic scenarios about the origin of the turbulent magnetic fields in the extragalactic: They are either generated from the evolution of primordial fields under the influence of structure formation, or by galactic plasma outflows (e.g. [3]). Based on different physical presumptions Hackstein et al. [2] $(\mathrm{H}+18)$ developed three different primordial models - with different seed field assumptions - and three different astrophysical models — with different energy budget assumptions of galactic halos. All of them are constrained by the local observational data. Nevertheless, the cumulative filling factors ${ }^{1}$ of the different EGMF models differ significantly: In principle, the primordial $\mathrm{H}+18$ models show the highest cumulative filling factors, followed by the astrophysical $\mathrm{H}+18$ models.

However, none of these magnetic field structures is capable of isotropizing the UHECR momenta of close-by - with distances of only a few Mpc - sources at the highest energies. Therefore, it is quite remarkable that one of the observed over-density regions, for energies $E \geq 50 \mathrm{EeV}$, coincides with the nearby radio galaxy Centaurus A. Further, a recent investigation [4] $(E+18)$ has shown that all of the observational characteristics of UHECRs can be explained by Centaurus A and Cygnus A. But thereto Cygnus A needs to provide a solar-like (light) CR contribution whose momenta get isotropized due to significant deflections by the EGMF. Otherwise, the dominant contribution up to $\sim 8 \mathrm{EeV}$ needs to be provided by a multitude of homogeneously and isotropically distributed sources. An interim solution, where only a few other, individual sources replace (or complement) the contribution by Cygnus A seems unlikely. On the one hand, Centaurus A and Cygnus A are by far the most brightest radio sources in the sky and on the other hand, a few individual source contributions still need appropriate deflections by the EGMF to account for the high degree of isotropy at energies $<8 \mathrm{EeV}$.

Thus, this work discusses only the limiting scenarios, where the UHECRs with energies $5 \mathrm{EeV} \lesssim E \lesssim 30 \mathrm{EeV}$ - hereafter referred to as low-energetic UHECRs - are provided rather by Cygnus A or the bulk of non-local radio galaxies. Additional details on this follow-up investigation can be found in a recent paper (E19) by the author [5].

\footnotetext{
${ }^{1}$ The filling factor indicates the fraction of the total volume filled with magnetic fields higher than a certain reference value.
} 


\section{UHECR contribution by Cygnus A}

The so-called inverse simulation setup, as recently introduced by E19, provides an efficient method to probe the impact of different EGMF models on the propagation of UHECRs. Hereby, the photohadronic interactions of the UHECRs with the cosmic microwave background and the extragalactic background light are taken into account. ${ }^{2}$ Supposing a CR ejection spectrum $\mathrm{d} N / \mathrm{d} E_{0} \propto$ $E_{0}^{-\alpha}$ from Cygnus A, the Fig. 1 shows the resulting mean of the CR deflection $\bar{\theta}$ and the trajectory length $\bar{l}_{\text {traj }}$ of the CR proton flux ${ }^{3}$ dependent on the observed energy $E$ for an initial spectral index $\alpha=2$. Since the impact of the magnetic fields on the CR increases with increasing charge number, more heavy nuclei show higher values of $\bar{\theta}$ and $\bar{l}_{\text {traj }}$, especially at low energies. But due to the decrease of the initial charge number by interactions, in particular at high energies these mean values hardly change. Thus, neither the initial type of CR nor the initial spectral index $\alpha$ have an
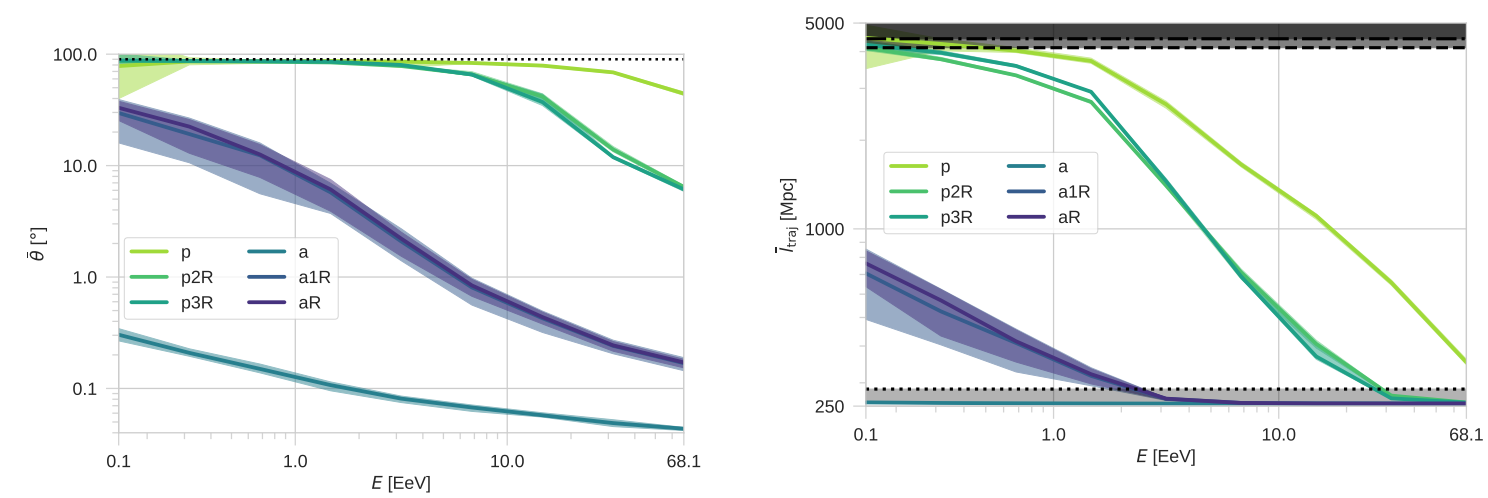

Figure 1: Mean deflections (left) and mean trajectory lengths (right) of a CR proton flux with $\alpha=2$ dependent on the observed energy for a source, like Cygnus A, at a distance of $255 \mathrm{Mpc}$. Here, different $\mathrm{H}+18$ EGMF models are used, where 'p'/ 'a' denotes primordial/ astrophysical models. The shaded band indicates the uncertainty due to the arbitrary spatial position of the sources. The dashed-dotted black line in the right figure indicates the Hubble distance, the dashed black line indicates the light travel distance of the first galaxy formations $(z=12)$ in the Universe, and the dotted black line indicates the maximal age of Cygnus A in terms of light travel distance.

effect on $\bar{\theta}$ and $\bar{l}_{\text {traj }}$ that is at the order of magnitude of the impact by the different EGMF models. In principle, the momenta of low-energetic UHECRs can be isotropized, in the case of one of the primordial $\mathrm{H}+18$ models, or show a significant degree of anisotropy, especially in the case of the astrophysical $\mathrm{H}+18$ model with an energy release of $5 \times 10^{58}$ erg per feedback episode starting from $z=4$. Since cosmological effects have not been taken into account within the simulation - apart from the redshift evolution of the photon background - the mean trajectory length of the CR flux can be compared to the light travel distance of some characteristic timescales of the source and the Universe, respectively. First galaxy formations in the Universe occur at a redshift $z \simeq 12$ yielding

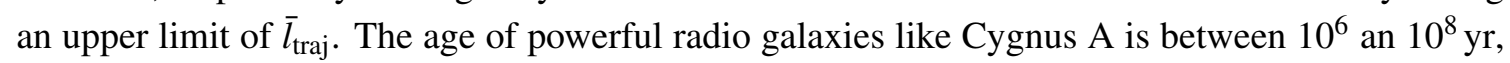

\footnotetext{
${ }^{2}$ For all details on the pure impact by the EGMF, i.e. without energy losses, on the total number of observed CRs, the interested reader is referred to E19.

${ }^{3}$ Note, that the solid angle dependence of the flux according to $\sin \theta$ needs to be taken into account, in contrast to the case where the total number of CRs is considered.
} 
e.g. [6], so that a correlation between the observed photon and CR flux signal is only feasible if the trajectory length of the $\mathrm{CR}$ is elongated less than about $30 \mathrm{Mpc}$ at most. Thus, if both types of messenger originate from the same source (Cygnus A), the CRs will be hardly deflected by the EGMF and the CR flux need to show a high degree of anisotropy in their arrival directions - in contrast to the observations. Otherwise, the observed CRs cannot originate from Cygnus A, as it is currently observed by photons.

\section{UHECR contribution by the bulk of radio galaxies}

An accurate estimate of UHECR contribution by the bulk of radio galaxies requires a unifying description of the physics of these sources. However, it is known since the classification from Fanaroff and Riley [7] that there are two major types of radio galaxies: FR-I RGs, in which the jets are terminating within the galactic environment on scales of a few kiloparsec, so that the brightness decreases with increasing distance from the central object; and FR-II RGs, where the jets extend on scales of $\gtrsim 100 \mathrm{kpc}$ deep into extragalactic space causing an increased brightness with distance. These different jet dynamics likely provide different energy budgets in CRs, as suggested by Godfrey and Shabala $[8,9]$. In addition, FR-I and FR-II sources are distributed significantly different in the Universe, as shown by their radio luminosity functions from Willott et al. [10]. Thus, the UHECR contribution from the bulk of FR-I and FR-II sources likely differ significantly. The crucial issue of this contribution is its spectral behavior, as already discussed by E+18. All details on the calculation of the resulting continuous source function are given by E19, so that only the main assumptions are briefly summarized in the following.

The energy density $Q_{\text {cr }}$ in CRs can be derived from the given radio luminosity $L_{151}$ at $151 \mathrm{MHz}$ by the heuristic correlation

$$
Q_{\mathrm{cr}}=\frac{g_{\mathrm{m}}}{1+k} Q_{\mathrm{jet}}=\frac{g_{\mathrm{m}}}{1+k} Q_{0}\left(\frac{L_{151}}{L_{p}}\right)^{\beta_{L}}
$$

where $g_{\mathrm{m}}$ denotes the fraction of jet energy found in leptonic and hadronic matter and the ratio of leptonic to hadronic energy density is given by $k$. In principle, $g_{\mathrm{m}}<1$ and in the case of a minimumenergy magnetic field this parameter yields $g_{\mathrm{m}} \simeq 4 / 7$ [11]. Unfortunately, most of the previously introduced quantities, including the normalization $Q_{0}$, the pivot luminosity $L_{p}$ as well as the slope index $\beta_{L}$, are poorly known, since the empirical methods that correlate the radio luminosity to the jet power are strongly affected by the distance dependence. However, Godfrey and Shabala argue that due to different distributions of the energy budget these quantities depend significantly on the FR type, suggesting that

$$
\beta_{L} \simeq \begin{cases}0.5 & \text { for FR-I } \\ 0.8 & \text { for FR-II } .\end{cases}
$$

However, in the case of powerful FR-I RGs a steeper slope in the range $0.5 \lesssim \beta_{L} \lesssim 0.8$ is expected. Still, the used correlation (3.1) lacks a fundamental relation to the different physics of the jet dynamics of FR-I and FR-II, which needs to investigated in more detail in future studies. But which consequences has the heuristic parameter $\beta_{L}$ on the UHECR contribution of the different FR types? 
To answer this question not only the total amount of CR energy according to Eq. (3.1) needs to be known, but also the maximal energy of the cosmic accelerators. Based on the common assumption of Bohm diffusion in the large scale structures of the jets, where the state of equilibrium is determined by the escape and acceleration timescale, the maximal rigidity yields [5]

$$
\hat{R} \equiv \frac{E_{\max }}{Z e}=g_{\text {acc }} \sqrt{\frac{\left(1-g_{\mathrm{m}}\right) Q_{\text {jet }}}{c}},
$$

with

$$
g_{\text {acc }}=\sqrt{\frac{8 \beta_{\text {sh }}^{2}}{f_{\text {diff }}^{2} \beta_{\text {jet }}}}
$$

Here, $1 \lesssim f_{\text {diff }} \lesssim 8$ encapsulates all details of the upstream and downstream plasma properties [12] in a strongly turbulent magnetic field for standard geometries [13]. So that in the case of the typical shock and jet velocities $\beta_{\text {sh }} \sim \beta_{\text {jet }} \sim 0.1$ in extended jets of radio galaxies the parameter values yield $0.01 \lesssim g_{\text {acc }} \lesssim 1$. Note, that only non-relativistic shocks are considered here, since relativistic ones are poor accelerators to EeV energies $[14,15]$. However, mildly relativistic, parallel shocks, where $0.2 \lesssim \beta_{\text {sh }} \lesssim 0.5$, are expected to be good UHECR accelerators [15], leading to $g_{\text {acc }} \sim 1$ at most.

Thus, there are three heuristic parameters $\left(Q_{0}, L_{p}\right.$ and $\left.\beta_{L}\right)$ that describe the correlation from radio luminosity to jet power, and three additional parameters $\left(k, g_{\mathrm{m}}\right.$ and $\left.g_{\text {acc }}\right)$ characterizing the resulting total CR power and maximal rigidity. In order to analyze the influence of these parameters a critical rigidity

$$
R_{\star}=g_{\mathrm{acc}} \sqrt{\frac{\left(1-g_{\mathrm{m}}\right) Q_{\star}}{c}}
$$

is introduced with

$$
Q_{\star}= \begin{cases}\left(\frac{4 \pi L_{L_{\star}}}{L_{p}}\right)^{\beta_{L}} Q_{0}, & \text { for FR-I, } \\ \left(\frac{4 \pi L_{\mathrm{h} \star}}{L_{p}}\right)^{\beta_{L}} Q_{0}, & \text { for FR-II },\end{cases}
$$

where $L_{1 \star}$ and $L_{\mathrm{h} \star}$ denote the break luminosity of the low-luminosity and the high-luminosity population, respectively [10]. It can be shown that at rigidities $R>R_{\star}$ the UHECR contribution provides a soft spectral behavior that cannot explain the hardening part of the observed spectrum of low-energetic UHECRs (see [5] for more details). Hence, only in the case of $R_{\star} \gtrsim 30 \mathrm{EV}$, the bulk of FR-I and FR-II sources, respectively, can provide the dominant UHECR contribution above the ankle. As shown in Fig. 2, FR-I sources need in the case of $\beta_{L} \lesssim 0.8$ a significantly higher minimal acceleration efficiency $g_{\text {acc }}^{\min }$ compared to FR-II sources to enable a proper spectral behavior above the ankle. Note that $g_{\mathrm{acc}}^{\min }$ decreases with decreasing $g_{\mathrm{m}}$, however, $g_{\mathrm{m}} \ll 4 / 7$ also reduces the total amount of ejected UHECR energy, so that the observed total energy flux can hardly be explained, especially in the case of FR-II RGs.

Analyzing the total UHECR energy in the hard part of the spectrum, i.e. at $6 \mathrm{EeV} \leq E \leq$ $20 \mathrm{EeV}$, the Fig. 3 exposes the fraction of the observed energy in the limiting case of a negligible lepton fraction, i.e. $k=0$, as well as an acceleration efficiency according to the conditions $R_{\star} \gtrsim$ $30 \mathrm{EV}$ and $g_{\text {acc }}=0.1$, in case $g_{\text {acc }}^{\min }<0.1$. So, the bulk of FR-I RGs needs neither a hard initial ejection spectrum, nor a high $\beta_{L}$ value to provide a significant amount of the observed UHECR 

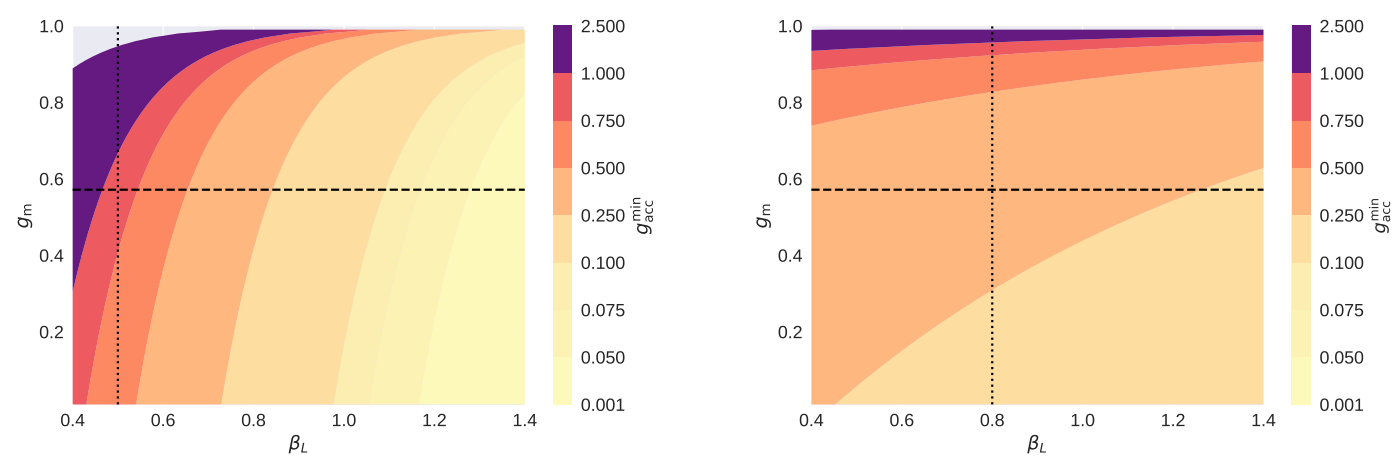

Figure 2: Minimal acceleration efficiency $g_{\text {acc }}^{\min }$ dependent on $g_{\mathrm{m}}$ and $\beta_{L}$ to obtain a critical rigidity $R_{\star} \geq$ $30 \mathrm{EV}$ in the case of the bulk of FR-I (left) and FR-II (right) RGs, respectively.

energy. Since the total UHECR energy roughly scales with $(1+k)^{-1}$, see [5], these parameter values slightly increase with increasing lepton fraction in order to provide the predominant part of the observed UHECR energy. In the case of FR-II RGs a hard ejection spectrum as well as $\beta_{L} \gtrsim 0.8$ and $g \gtrsim 4 / 7$ is needed to provide more than $50 \%$ of the observed total UHECR energy. In contrast to the case of FR-I RGs, the chosen parametrization of the radio luminosity functions given by Willott et al. [10] has a huge impact on the resulting amount of UHECRs at Earth. Here, the radio luminosity functions in the case of a flat cosmology $\left(\Omega_{\mathrm{M}}=1\right)$ yields the most UHECR energy by FR-II RGs, in particular for the parameter model A (see [10] for more details), where in the case of $\alpha=0.5, g_{\mathrm{m}} \simeq 1$ and $\beta_{L} \simeq 1.4$ about $90 \%$ of the observed CR energy can be provided by FR-II RGs. As the continuous source function of FR-I and FR-II RGs is proportional to $\left(R / R_{\star}\right)^{-\alpha}$ for $R \ll R_{\star}$ as shown by E19, the total UHECR energy also increases for a higher normalization $Q_{0}$ according to $Q_{0}^{\alpha / 2}$. Still a significant UHECR contribution by FR-II RGs can only be obtained at the extreme end of the parameter space.

Note that the total UHECR energy only gives a necessary criterion, as the spectral behavior still needs to fit to the observations. Therefore, the initial spectral index $\alpha$ needs to be adjusted, but hereby the additional UHECR contribution at $E>20 \mathrm{EeV}$ needs to be taken into account, which is beyond the scope of this work.

\section{Conclusions}

UHECRs above the so-called GZK-cutoff energy of about $50 \mathrm{EeV}$ need to be explained by local sources, i.e. at a distance $\lesssim 50 \mathrm{Mpc}$. Here, the close-by FR-I RG Centaurus A shows great potential — from theoretical and observational sight — of being the dominate source. However, the change of the chemical composition and the arrival directions indicate that low-energetic UHECRs most likely originate somewhere else. A prominent source at these energies is the FR-II RG Cygnus A, due to its extreme brightness in the radio band.

It is shown that the primordial $\mathrm{H}+18$ models are capable of an isotropization of the lowenergetic UHECRs from Cygnus A, however, the corresponding propagation time exceeds the light travel time by a multitude of the source age. At energies above the ankle, only the astrophysical 

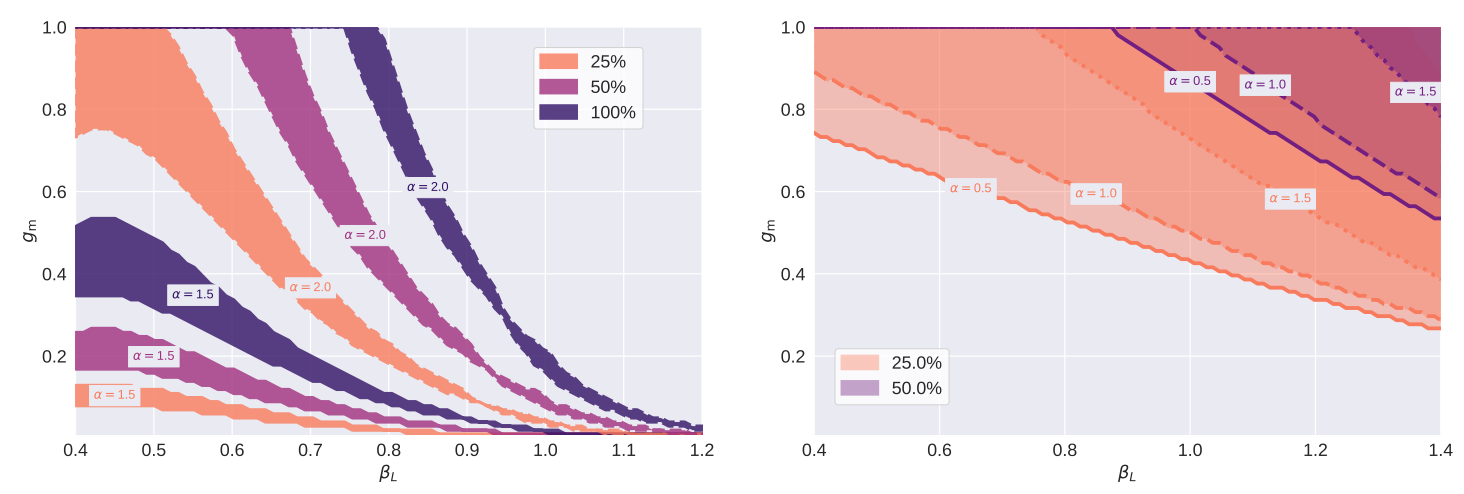

Figure 3: Total UHECR energy contribution between $6 \mathrm{EeV}$ and $20 \mathrm{EeV}$ dependent on $g_{\mathrm{m}}$ and $\beta_{L}$ with $g_{\text {acc }} \leq 0.1$ by the bulk of FR-I (left) and FR-II (right) RGs, respectively.

$\mathrm{H}+18$ models yield a delay that is smaller than the source age, but the mean deflection is at the order of a few degrees at most. Thus, either the photons from Cygnus A cannot be correlated to the CRs, which annuls the reason for choosing Cygnus A in the first place, or the contribution from Cygnus A disagrees with the observed arrival directions. ${ }^{4}$ Therefore, the low-energetic UHECR contribution seems more likely to be provided by a multitude of sources, in particular in the case of a weak EGMF strength according to the astrophysical H+18 model.

The low-energetic UHECR contribution by the bulk of FR-I and FR-II sources depends on several parameters - resulting from the radio luminosity function, the radio-to-CR correlation and the acceleration process - which are not well constrained by observations. Based on the assumption of a dominant low-energetic UHECR contribution, it is shown that an appropriate spectral behavior can be provided by FR-II RGs for a huge range of parameters, while FR-I RGs need a high acceleration efficiency or a radio-to-CR correlation index $\beta_{L}>0.5$. But FR-I RGs most likely provide the necessary total amount of low-energetic UHECR energy, whereas FR-II RGs would need a significantly higher normalization $Q_{0}$ of the radio-to-CR correlation, as well as a negligible lepton fraction, i.e. $k \simeq 0$, and $g_{\mathrm{m}} \gtrsim 4 / 7$. In total, only the bulk of FR-I sources shows great potential of being the dominant contributor to low-energetic UHECRs, that needs to be investigated in more detail in future investigations.

\section{References}

[1] K. Dolag et al. "Constrained Simulations of the Magnetic Field in the Local Universe and the Propagation of Ultrahigh Energy Cosmic Rays". In: Journal of Cosmology and Astroparticle Physics 1 (2005), p. 9.

\footnotetext{
${ }^{4}$ Note that the impact of the Galactic magnetic field has not been taken into account. However, the commonly used magnetic field model by Jansson and Farrar [16] can only yield the necessary large scale deflections of light UHECRs at $8 \mathrm{EeV}$ in the case of a strong random magnetic field component — which is most likely by a factor of 1.7 to 3 too strong [17] — with a large (>100 pc) coherence length [18].
} 
[2] S. Hackstein et al. "Simulations of ultra-high energy cosmic rays in the local Universe and the origin of cosmic magnetic fields". In: Monthly Notices of the Royal Astronomical Society 475.2 (2018), pp. 2519-2529.

[3] R. M. Kulsrud and E. G. Zweibel. "On the origin of cosmic magnetic fields". In: Reports on Progress in Physics 71.4 (2008), p. 046901.

[4] B. Eichmann et al. "Ultra-high-energy cosmic rays from radio galaxies". In: Journal of Cosmology and Astroparticle Physics 2018.02 (2018), p. 036.

[5] B. Eichmann. "High Energy Cosmic Rays from Fanaroff-Riley radio galaxies". In: Journal of Cosmology and Astroparticle Physics 2019.05 (2019), p. 009.

[6] C. L. Carilli and P. D. Barthel. "Cygnus A". In: Astron. Astrophys. Rev. 7.1 (1996), pp. 1-54.

[7] B. L. Fanaroff and J. M. Riley. "The Morphology of Extragalactic Radio Sources of High and Low Luminosity”. In: Mon. Not. Roy. Astron. Soc. 167 (1974), 31P-36P.

[8] L. E. H. Godfrey and S. S. Shabala. "AGN Jet Kinetic Power and the Energy Budget of Radio Galaxy Lobes”. In: The Astrophysical Journal 767.1 (2013), p. 12.

[9] L. E. H. Godfrey and S. S. Shabala. "Mutual distance dependence drives the observed jetpower-radio-luminosity scaling relations in radio galaxies". In: Monthly Notices of the Royal Astronomical Society 456.2 (2016), pp. 1172-1184.

[10] C. J. Willott et al. "The radio luminosity function from the low-frequency 3CRR, 6CE and 7CRS complete samples”. In: Monthly Notices of the Royal Astronomical Society 322.3 (2001), pp. 536-552.

[11] A. G. Pacholczyk. Radio Astrophysics. Nonthermal Processes in Galactic and Extragalactic Sources. W. H. Freeman \& Co Ltd, San Francisco, 1970.

[12] A. R. Bell et al. "Cosmic-ray acceleration and escape from supernova remnants". In: Monthly Notices of the Royal Astronomical Society 431.1 (2013), pp. 415-429.

[13] L. Oc. Drury. "An Introduction to the Theory of Diffusive Shock Acceleration of Energetic Particles in Tenuous Plasmas”. In: Rept. Prog. Phys. 46 (1983), pp. 973-1027.

[14] A. R. Bell and B. Reville. "On the maximum energy of shock-accelerated cosmic rays at ultra-relativistic shocks". In: Monthly Notices of the Royal Astronomical Society 439.2 (2014), pp. 2050-2059.

[15] A. R. Bell et al. "Cosmic-ray acceleration by relativistic shocks: limits and estimates". In: Monthly Notices of the Royal Astronomical Society 473.2 (2017), pp. 2364-2371.

[16] R. Jansson and G. R. Farrar. "A New Model of the Galactic Magnetic Field”. In: The Astrophysical Journal 757 (2012), p. 14.

[17] M. C. Beck et al. "New constraints on modelling the random magnetic field of the MW". In: Journal of Cosmology and Astroparticle Physics 2016.05 (2016), pp. 056-056.

[18] G. R. Farrar and M. S. Sutherland. "Deflections of UHECRs in the Galactic magnetic field". In: Journal of Cosmology and Astroparticle Physics 2019.05 (2019), pp. 004-004. 